\title{
CONSIDERATIONS SUR L'AVANCEMENT DANS LES CARRIERES PROCURATORIENNES EQUESTRES
}

Par

\section{S. DEMOUGIN}

Le point de départ des réflexions que l'on peut faire sur le déroulement des carrières procuratoriennes équestres ${ }^{1}$ vient d'une très célèbre lettre de Fronton ${ }^{2}$, adressée à Marc Aurèle qui n'était encore alors qu'héritier désigné, et lui recommandant, pour l'octroi d'une procuratèle, l'affranchi impérial Aridelus dont il rappelle la demande : petit nunc procurationem ex forma suo loco ac iusto tempore. H.-G. Pflaum, qui a naturellement repris l'étude de ce document dans son livre sur les procurateurs de rang équestre ${ }^{3}$, conclut son analyse $d u$ document dans les termes suivants : " les mots... attestent en effet explicitement l'existence de la hiérarchie romaine pour les procuratèles affranchies. Il ne sera pas trop téméraire de supposer des réglements analogues pour les emplois équestres"4. Cette existence d'un réglement, et même d'un réglement annuel est confirmée, pour le maître des études procuratoriennes, par deux témoignages: tout d'abord, celui venant d'une notation de Suétone, parlant, dans la vie de Domitien, de l'ordinatio proxima $a^{5}$ qui a vu la promotion de Mettius Rufus comme préfet $\mathrm{d}^{\prime}$ Egypte $^{6}$; il est possible qu'alors le "mouvement administratif", d'après l'expression de H.-G. Pflaum, se plaçait en novembre', après la rentrée du sénat. La seconde occurence est aussi à chercher dans les lettres de Fronton, dans le dossier consacré à Appien; on y voit tout d'abord le maître de Marc Aurèle solliciter Antonin le Pieux en faveur d'Appien ${ }^{8}$ et lui rappeler que c'est la troisième année qu'il prie le souverain d'accorder une procuratèle à son ami. Donc, en conclusion de l'examen de tous ces témoignages, H.-G. Pflaum concluait à l'existence d'une système de promotion annuelle, fait à date fixe, et se fondant sur une hiérarchie fixe des postes, système défini par la phrase de Fronton, qui "s'avère comme la grande règle de la hiérarchie romaine"9 ; mais il tempérait aussi la rigueur de sa position en

\footnotetext{
${ }^{1}$ Les éléments sur lesquels se fonde cet article sont issus de la banque de données PROC, en cours d'élaboration dans l'UMR 8585, Paris.

${ }^{2}$ Ad Marcum Caes. 5.52.

${ }^{3}$ H.-G. Pflaum, Les procurateurs équestres sous le Haut-empire romain (Paris 1950).

${ }^{4}$ H.-G. Pflaum 1950, op.cit. (n.3), 199.

${ }^{5}$ Suétone, Domitianus 4.

${ }^{6}$ H.-G. Pflaum 1950, op.cit. (n.3), 204.

${ }^{7} K V W^{2}, 442-445$.

${ }^{8}$ Ad Antoninum Pium 10.

${ }^{9}$ H.-G. Pflaum 1950, op.cit. (n.3), 296.
} 
rappelant que " le côté individuel et particulier de chaque promotion ne saurait être négligé et cela d'autant moins que la faveur impériale est un facteur indiscutable... la rigueur des réglements... a été heureusement tempérée par la souplesse de leur application et le choix de l'empereur a, en dernière instance prévalu sur l'ancienneté la mieux établie"10.

Cette conception, assez rigide il faut bien le reconnaître, se doublait aussi d'un autre postulat ; la spécialisation des postes et des hommes. Ainsi, H.G. Pflaum considérait qu' un certain nombre de procurateurs était choisi pour des types de postes bien précis, en raison de compétences particulières qu'ils possédaient, et dont le plus bel exemple reste celui des aduocati fisci. De même, il s'appuyait sur la différence fondamentale qu'il croyait déceler dans le recrutement des procurateurs, entre procurateurs latinophones et procurateurs hellénophones, ceux-ci tendant à ne parcourir leur carrière que dans les provinces où le grec était la seule langue pratiquée. Ainsi, il en arrivait à une conception relativement rigide de l'exercice des fonctions équestres : celles-ci étaient non seulement ordonnées selon une stricte hiérarchie due aux salaires alloués aux procurateurs, mais encore elles étaient octroyées à date fixe, dans un mouvement annuel, et elles étaient données aux fonctionnaires suivant leurs propres capacités et leurs compétences personnelles.

La conception de ce système administratif, dont on pourrait trouver de lointains héritiers dans tous les siècles postérieurs, fit l'objet d'attaques, à partir du moment où l'historiographie contemporaine revint sur deux éléments fondamentaux, liés de plus l'un à l'autre : le rôle de l'empereur d'une part" ${ }^{11}$, et l'exercice du patronage qui connut un regain d'intérêt, entre 1970 et 1990 ; tous les types de recommandation ${ }^{12}$ furent à nouveau examinés, pour la République et pour l'Empire ${ }^{13}$. En ce qui concerne les procurateurs, s'il restait indéniable que la hiérarchisation salariale gouvernait le déroulement du cursus équestre, en revanche furent mis en doute le principe d'un avancement et d'un recrutement annuel, ainsi que celui de la spécialisation. Les conceptions de H.G. Pflaum avaient déjà été critiquées par F. Millar ${ }^{14}$ et son interprétation de la

\footnotetext{
${ }^{10}$ Pflaum 1950, op.cit. (n.3), 296.

${ }^{11}$ Illustré par le célèbre livre de F. Millar, The Emperor in the Roman World (Londres 1977).

${ }^{12}$ Voir ainsi H. Cotton, Documentary Letters of Recommendation in Latin from the Roman Empire (Königstein 1981).

${ }^{13}$ Voir, par exemple, E. Deniaux, Clientèles et pouvoir à l'époque de Cicéron (Rome 1993) et, pour le patronat judiciaire J.M. David, Le patronat judiciaire au dernier siècle de la République (Rome 1992). Pour un cas particulier sous l'Empire, voir $\mathrm{H}$. Cotton, 'Military Tribune and the Exercise of Patronage', Chiron 11 (1981), 229-239.

${ }^{14}$ Voir le compte-rendu des Carrières procuratoriennes équestres par F. Millar, JRS 53 (1963), qui concluait à l'inexistence de règles.
} 
lettre de Fronton en faveur d'Aridelus, que j'ai présentée plus haut, fut repoussée par E. Champlin ${ }^{15}$, préférant interpréter autrement le passage $e x$ forma, suo loco, ac iusto tempore, et le comprenant comme "in the proper manner, on his own behalf, and at the proper time"16. A son tour, R.P. Saller ${ }^{17}$, est revenu sur ces problèmes de promotion ${ }^{18}$, en privilégiant trois domaines : "seniority, promising careers et personel criteria" ; il en conclut que ni l'ancienneté ni la spécialisation, des facteurs détectables dans les simples listes de procuratèles, ne permettent d'expliquer de manière satisfaisante le déroulement des cursus, contrairement aux facteurs personnels, surtout le patronage dont l'influence est décisive sur les décisions de l'empereur' ${ }^{19}$. R.P. Saller a maintenu ce point de vue dans son livre sur le patronat ${ }^{20}$. Mais le coup de grâce a été asséné - ou aurait voulu être asséné - par P. Brunt, en deux étapes. Tout d'abord dans son étude sur les préfets d'Egypte ${ }^{21}$, puis dans son enquête sur les relations entre le prince et les chevaliers ${ }^{22}$,il met en relief la faible fiabilité des données documentaires sur lesquelles H.-G. Pflaum a bâti son système, et fonde sa démonstration sur plusieurs points qu'on va reprendre ici. Premièrement, P.A. Brunt insiste sur la fonctionnalité du service militaire, qui, pour les futurs procurateurs, est plus long que celui des jeunes membres de l'ordre sénatorial et amène donc les officiers équestres à être bien préparés pour l'administration militaire, mais beaucoup moins armés pour les charges civiles. Il serait trop long d'argumenter ici sur les buts et les conséquences des militiae. On doit faire cependant quelques remarques : tous les officiers n'accomplissent pas les milices réglementaires, et se contentent d'une seule ou de deux étapes, sans que l'on puisse toujours attribuer cette situation à la relative faiblesse des postes disponibles, mise en évidence par les études d'E. Birley, et sans que cela constitue un handicap pour une carrière future. Il est même des procurateurs

\footnotetext{
${ }^{15}$ E. Champlin, Fronto and the Antonine Rome (Cambridge Mass.-Londres 1980), 102.

${ }^{16}$ Champlin 1980, op.cit. (n. 15), 102, n. 53, note que le texte de Fronton est loin d'être sûr ; mais dans ses deux édition successives, $M$. van den Hout a maintenu le texte sous la forme traditionnelle ; dans son commentaire à Fronton (Amsterdam 1999), 211 il commente ainsi ce difficile passage : ex forma "according to the rules" ; suo loco ac iusto tempore "in view of the rank (he holds now) and the requisite seniority", sans renvoyer aucunement à l'oeuvre de H.-G. Pflaum, ni aux ouvrages des spécialistes des affranchis impériaux, P.R.C. Weaver et G. Boulvert.

${ }^{17}$ R.P. Saller, 'Promotion and Patronage in Equestrian careers', Journal of Roman Studies 70 (1980), 44-63.

${ }^{18}$ Saller 1980, l. c. (n.18), 53, reconnaît que les deux interprétations de la phrase de Fronton, celle de H.-G. Pflaum, et celle de E. Champlin sont acceptables.

${ }^{19}$ Idem., ibid., 57-58.

${ }^{20}$ Personal Patronage under the Early Empire (Oxford 1982).

${ }^{21}$ P.A. Brunt, 'The Administrators of Roman Egypt', Journal of Roman Studies 65 (1975), 124-137.

${ }^{22}$ Idem, 'Princeps and Equites', Journal of Roman Studies 73 (1983), $42-75$.
} 
faisant état d'un service militaire allégé, qui ne les empêche pas de faire une brillante carrière. Pour n'en citer qu'un exemple pour ne pas lasser le lecteur, rappelons la carrière de Sex. Attius Suburanus Aemilianus ${ }^{23}$, qui après une préfecture des ouvriers et une préfecture d'aile, ne reçut pas moins de sept procuratèles, dans le sillage, il est vrai, de puissants protecteurs, Vibius Crispus et Iulius Ursus ${ }^{24}$. A cet égard, on peut relever les contradictions qui émanent de nos sources elles-mêmes : prenons ainsi le préfet du prétoire de Néron, Burrus $^{25}$, honoré à Vaison par ses compatriotes. On ne relève dans son cursus que la simple mention de trib(unus) mil(itum), tandis que Tacite nous parle de son egregia militaris fama ${ }^{26}$. Il faudrait faire la part aussi de l'octroi des milices honoraires, créées par Claude et annoncées par l'institution fugace des tribuni militum a populo par Auguste. On peut se demander si, dans certains cas, la simple mention d'un grade, non accolé du nom de l'unité commandée ne nous signale pas un détenteur de ces milices honoraires, qui n'empêchent pas ensuite de faire une carrière civile.

Ensuite, P.A. Brunt insiste sur l'absence de spécialisation des procurateurs, qui deviennent en quelque sorte des amateurs éclairés, ne possédant pas de véritables compétences. In ne faut pourtant pas oublier que l'éducation aristocratique comportait une formation intellectuelle, où le droit n'était pas négligé. Il est vrai aussi que l'une des caractéristiques des hauts fonctionnaires est de pouvoir passer d'une sphère de responsabilité à l'autre; les exemples en seraient innombrables dans le monde moderne. Il n'empêche qu'on ne peut nier que dans certains cas, il a été fait appel à de vrais spécialistes, en particulier en matière financière et en matière juridique. Pour ne prendre que ce dernier domaine, la participation des iurisperiti aux sessions du conseil impérial est bien connu. Dans ces dernières années, on a vu émerger ainsi quelques figures, comme celle de Q. Baebius Modestus, allectus inter consiliarios de Caracalla et Geta, et devenu immédiatement après préfetprocurateur de Sardaigne ${ }^{27}$, surtout celle de M. Cn. Licinius Rufinus, fort célèbre juriste, dont la carrière n'était connue que dans sa partie sénatoriale, et qui s'avère maintenant être titulaire d'une riche carrière équestre, passée, dans

\footnotetext{
${ }^{23}$ H.-G. Pflaum, Les carrières procuratoriennes équestres (Paris 1960-1), $\mathrm{n}^{\circ} .56$.

${ }^{24}$ IGLS 6,2785.

${ }^{25}$ Pflaum 1960-1, op.cit. (n. 23), $n^{\circ} .13$.

${ }^{26}$ CIL XII 5842 (D. 1321) ; Tacite Ann. 12,42.

${ }^{27}$ P. Benito Serra et G. Bacco, 'Forum Traiani : il contesto termale e l'indagine archeologica di scavo', L'Africa romana 12 (1996), 1244.
} 
sa partie connue, dans le conseil impérial et dans les bureaux palatins $\mathrm{s}^{28}$. Il vaut mieux considérer que l'administration romaine est pragmatique: il arrive que soient nommés effectivement des non-professionnels, mais, en général, les compétences sont utilisées. En ce qui concerne l'expérience acquise par des responsabilités administratives exercées au niveau municipal, on tombera d'accord avec P.A. Brunt que la rédaction des carrières comportant deux volets, procuratorien et municipal, restent difficiles à interpréter; E. Birley s'était heurté au même obstacle avec les cursus des officiers équestres comportant des magistratures et des honneurs locaux ${ }^{29}$. Effectivement le rôle joué par les chevaliers dans les collectivités locales ne leur était pas d'une grande aide pour obtenir des postes dans l'administration impériale; à un autre niveau, il en va d'ailleurs de même pour les grands-prêtres provinciaux. Mais nous manquons d'éléments sûrs pour considérer que l'entrée dans la carrière municipale se faisait, pour beaucoup de chevaliers, après l'accomplissement des milices équestres, qui constituait le premier pas vers la carrière civile. Un phénomène est à mettre en valeur: le fait que peu de hauts fonctionnaires équestres s'acquittent d'une carrière municipale ${ }^{30}$, même s'ils acceptent des patronats qui restent des honneurs bien spécifiques, dont il n'y a pas à tenir compte dans la discussion. Cette tendance est confirmée par la documentation nouvelle qui sera présentée plus bas: seuls trois procurateurs ont suivi le cursus municipal, T. Mercello Persinus Marius ${ }^{31}$, P. Rutilius Bassus ${ }^{32}$, et C.? Sallius? Proculus? ${ }^{33}$.

Quant à la durée des procuratèles, je crois qu'il faut rester sage: $\mathrm{O}$. Hirschfeld, avec bon sens, observait que nous manquions de données fiables à ce sujet ${ }^{34}$. En revanche, H.-G. Pflaum et P.A. Brunt, se fondant tous deux sur des données statistiques, se sont opposés à ce sujet, l'un pensant que deux années séparaient la tenue d'une fonction procuratorienne, l'autre tenant pour trois années, en se fondant sur la liste des préfets d'Egypte d'une part, et celle des épistratèges, d'autre part. On fera les réserves qui s'imposent pour les préfets d'Egypte, vice-rois, fonctionnaires d'autorité, nommés et révocables ad

\footnotetext{
${ }^{28} \mathrm{P}$. Herrmann, 'Die Karriere eines proeminenten Jurist aus Thyateira', Tychè 12 (1998), 111-123; F. Millar 'The Greek East and Rome: the Dossier of M. Cn. Licinius Rufinus', Journal of Roman Studies 89 (1999), 90-108.

${ }^{29}$ E. Birley, The Equestrian Officers of the Roman Army', Durham University Journal (1949), 8-19= The Roman Army, 147-164.

${ }^{30}$ Voir les relevés de Brunt 1975, l. c. (n.21), 50.

${ }^{31}$ A. Caballos Rufino, Kolaios 4 (1995), 294-295.

${ }^{32}$ IvE 1538 ; IvE 2038, cf. W. Eck, Zeitschrift für Papyrologie und Epigraphik 117 (1997), 107-128.

${ }^{33}$ SI 9, 82 no. 31 (AE 1983, 325) Amiternum.

${ }^{34} \mathrm{KVW} W^{2} 445-447$.
} 
nutum $^{35}$. En pareil cas, il faut renoncer à l'établissement d'une règle. Et cette souplesse apparaît très clairement dans les périodes de crise, quand on constate la rapide rotation des responsables équestres ${ }^{36}$. Par ailleurs, il semble assez évident, comme le rappelle P.A. Brunt, que l'avancement des jeunes fonctionnaires équestres dépendait de leurs appuis politiques: j'en ai cité un exemple plus haut, avec Attius Suburanus, qui étale complaisamment les noms de ses protecteurs. On doit cependant élargir ces pratiques à l'ensemble du corps social, même si on le cerne mieux pour les niveaux les plus élevés de la société, et plus particulièrement pour les membres de l'ordre équestre ${ }^{37}$. En revanche, on ne peut affirmer que seule, dans les bureaux, la familia Caesaris possède l'expérience professionnelle, et comparer cette situation avec celle des centurions dans l'armée; en effet, le statut des affranchis est parfaitement clair, contrairement à celui des officiers subalternes, issus soit du rang, soit de l'ordre équestre; ces derniers, venus de l'une des deux aristocraties, jouissent d'avantages considérables dans l'avancement et la carrière ultérieure.

On tombera, en revanche, d'accord avec les critiques de P.A. Brunt sur les conceptions erronées $\mathrm{d}^{\prime} \mathrm{A}$. $\mathrm{Stein}^{38}$ présentant encore l'ordre équestre républicain comme un ensemble de "manieurs d'argent", conception dont les historiens majeurs de ce siècle ont fait justice ${ }^{39}$. Cependant, pour comprendre le déroulement de la carrière équestre, il faut trouver un juste équilibre entre deux conceptions, l'extrême rigueur dans le service civil, ou le complet laxisme dans les choix et les promotions des fonctionnaires. A cet égard, il faut prendre garde aux constructions trop théoriques: ainsi, la reconstruction de la hiérarchie des grandes préfectures, vigiles, annone, Egypte, prétoire, ne doit pas faire oublier que nous ne connaissons qu'un seul chevalier passé par les quatre degrés, C. Tettius Africanus Cassianus Priscus ${ }^{40}$, "ce qui constitue un cumul sans autre exemple dans les carrières supérieures équestres" ${ }^{\prime 41}$.

\footnotetext{
${ }^{35}$ Voir les considérations de R. Sablayrolles, 'Les grandes préfectures équestres', L'ordre équestre, histoire d'une aristocratie (Bruxelles-Louvain 1995 [Rome, 1999]), 354.

${ }^{36}$ Voir par exemple le cursus de M. Aquilius Felix, Pflaum 1960-1, op.cit. (n.23), n 25.

${ }^{37} \mathrm{Cf}$. H. Devijver, 'Les relations sociales des chevaliers romains', L'ordre équestre, histoire d'une aristocratie (Bruxelles-Louvain 1995 [Rome 1999]), 237-269.

${ }^{38}$ A. Stein, Der römische Ritterstand (Munich, 1927).

${ }^{39} \mathrm{Cl}$. Nicolet, L'ordre équestre à l'époque républicaine (Paris 1965).

${ }^{40}$ CIL XI $5382=$ I. Assisium 17 ; PIR ${ }^{2}$ C 570.

${ }^{41}$ Sablayrolles 1999, l. c. (n.35), 369, avec la note 27.
} 
Il convient d'examiner la documentation nouvelle, publiée depuis une vingtaine d'années, pour voir si elle nous a permis d'améliorer notre connaissance de la forma, du locus et du tempus de la carrière procuratorienne. Une enquête attentive fait connaître l'existence de 120 nouveaux procurateurs ${ }^{42}$, dont sept inédits, dont la déontologie nous interdira de parler ici. La grande majorité d'entre eux, malheureusement, n'a exercé, du moins à notre connaissance qu'une seule fonction; ainsi en va-t-il des épistratèges, dont les listes se sont augmentées et complétées depuis une vingtaine d'années ${ }^{43}$. Ces fonctionnaires seront donc exclus de notre discussion ; en effet, pour mener la contre-épreuve, il faut impérativement adopter les mêmes critères que H.-G. Pflaum, c'est-àdire ne tenir compte que des cursus comprenant au moins deux fonctions procuratoriennes.

Prenons d'abord la forma. Hors les textes canoniques que nous avons cités plus haut, il faut verser au dossier une nouvelle pièce, la "lettre de promotion" envoyée par Marc Aurèle à son procurateur, Q. Domitius Marsianus $^{44}$, pour l'élever à la ducenariae procurationis splendor. L'empereur ajoute qu'il a saisi l'occasion:"utor opportunitate quae nunc [o]btegit". H.-G. Pflaum, qui, le premier, s'est attaché à ce document, a insisté sur le fait que "le moment favorable dont Marc-Aurèle profite pour promouvoir Marsianus lui est échu par hasard"45. Cependant, l'insistance mise par l'empereur sur la "chance" qui a fait que le poste de procurateur ducénaire du patrimoine de Narbonnaise était libre constitue un autre indicateur: d'une part la nomination n'était pas attendue, ce qui veut dire que le poste a été libéré de manière brutale; d'autre part, il est évident qu'il y a d'autres nominations qui ne sont pas dues au hasard. Je crois, là encore, qu'il ne faut pas être systématique. Les indices que nous avons rassemblés plus haut militent en faveur d'une ordinatio annuelle, fondée sur un tableau d'avancement cohérent, dont les postes sont distribués habituellement selon le principe d'ancienneté. Cependant, il ne faudrait pas attribuer au système romain une rigidité qu'il n'a pas : toutes les nominations et

\footnotetext{
${ }^{42}$ Il faut sans doute, en fait, en ajouter un autre, si l'on considère que le Didius Secundus, destinataire d'un rescrit de Trajan, Dig., 48.22.1 n'est pas un proconsul d'Asie, mais plutôt un procurateur, comme le suggère W. Eck, Chiron 12 (1982), 337.

${ }^{43}$ Signalons ici l'ouvrage de J.D. Thomas, The Roman Epistrategos (Opladen 1982), dont on espère que l'auteur procurera une seconde édition..

${ }^{44}$ AE 1962, 183, Bulla Regia.

${ }^{45}$ H.-G. Pflaum, 'Une lettre de promotion de l'empereur Marc-aurèle pour un procurateur ducénaire de Gaule Narbonnaise', Bonner Jahrbücher 171 (1971), 349-366 = La Gaule et l'Empire romain, 12-29.
} 
promotions sont aux mains de l'empereur, et donc il lui reste la latitude de bouleverser la hiérarchie, de procéder à des promotions parfois inouïes et d'intervenir en dehors de la distribution annuelle. Nous avons des traces de ces pratiques surtout dans les périodes troublées; en témoigne la carrière étonnante de Cassius Seuerus ${ }^{46}$, passé de la préfecture de cohorte aux procuratèles de Macédoine et de Thrace, puis promu à la préfecture de la flotte de Ravenne, qu'il ne faut peut-être attribuer aux débuts du règne de Septime Sévère, mais qui témoigne d'un manquement aux règles en usage.

Cependant, on ne peut que constater l'existence d'un schéma de carrière classique et court, comprenant, après les milices équestres, trois procuratèles : la première de rang sexagénaire, la seconde de rang centenaire, et la troisième de rang ducénaire. On le verra, par exemple, avec Ti. Claudius Antoninus ${ }^{47}$, qui, après avoir exercé les trois milices réglementaire, géra le vingtième des héritages des Gaules et des Germanies, puis les finances de la Macédoine, province du peuple romain, enfin celles de la Bretagne, province relevant de l'empereur. On pourrait citer aussi un procurateur anonyme de $\mathrm{Nyon}^{48}$ au cursus vraisemblablement identique ${ }^{49}$ dans son déroulement.

Dans la nouvelle documentation, on ne peut mentionner, pour notre propos, que les carrières qui présentent plusieurs procuratèles, ce qui va permettre de discuter justement de la pertinence des modalités de la promotion; mais on devra signaler les cas douteux, comme celui justement de Cassius Seuerus, dont je viens de parler, et dont les fonctions en Thrace et en Macédoine ont été considérées, sans doute à tort, comme conjointes ${ }^{50}$. Il y a donc à considérer les carrières où nous trouvons deux procuratèles : celles de [Volusius ? Martialis] ${ }^{51}$, de M. Rutilius Cosinius G[-]us ${ }^{52}$. On y ajoutera celle de T. Flauius Geminus, récemment publiée par Fr. Bérard ${ }^{53}$. Quant aux détenteurs de trois procuratèles, ils sont relativement plus nombreux : T. Petronius Priscus $^{54}$, a libellis d'Hadrien, ou celle de l'anonyme de Lyon ${ }^{55}$, AE, 1986, 127,

\footnotetext{
${ }^{46}$ SEG 35 (1985), 829 = SEG 43 (1382), Philippolis.

${ }^{47}$ L'inscription qui mentionne cet officier équestre n'a pas encore reçu de commentaire savant; on en trouvera le texte dans PME, Supp. 2, C 118 bis ; cf. S. Demougin, CCG 9 (1998), 224-225.

${ }^{48}$ AE 1996, 1115.

${ }^{49}$ Cette carrière sera étudiée à nouveau par $\mathrm{S}$. Demougin et $\mathrm{X}$. Loriot dans un article à paraître.

${ }^{50}$ Comme l'ont voulu les deux premiers commentateurs, L. Botusanova et D. Boteva.

${ }^{51}$ RMD 3,186 ; RMD 3,177.

${ }^{52}$ AE 1993, 1005, Italica ; voir les commentaires d'A. Caballos Rufino, Kolaios (1995), 333 no. 37 et de W. Eck, Zeitschrift für Papyrologie und Epigraphik 124 (1999), 228-230.

${ }^{53}$ AE 1995, 201 ; voir aussi PME Supp. 2, C 49 a.

${ }^{54}$ AE 1993, 1477, Ephesus.

${ }^{55}$ AE 1986, 127.
} 
dont la carrière a été remise sur pied par M. Christol ${ }^{56}$ puis C.? Sallius? Proculus $^{57}$ ou un anonyme de Gerasa ${ }^{58}$. On peut continuer par énumérer le nombre de procuratèles obtenues, ce qui est aussi un indicateur de la longévité tout aussi bien personnelle et administrative : ainsi, pour le niveau des quatre offices bureaucratiques obtenus, on citera - et toujours dans l'ordre chronologique - un primipile bis resté anonyme de Rome, dont le cursus compliqué a été présenté par S. Priuli ${ }^{59}$, Sex. Cornelius Repentinus dont une version du cursus probable a été proposée par G. Camodeca ${ }^{60}$, et enfin M. Domitius Tertius, dont le dernier poste connu est la procuratèle-gouvernement de Sardaigne ${ }^{61}$.

Nos dernières catégories permettent de mieux comprendre le fonctionnement de la carrière, vu le nombre de postes qui ont été conservés pour chaque individu: ainsi on observera les parcours d'un anonyme d'Ephèse ${ }^{62}$, et de C. Seruilius Diodorus dont l'extraordinaire dossier de donation vient d'être publiée ${ }^{63}$, et qui a déjà suscité des commentaires ${ }^{64}$; tous deux ont obtenu cinq procuratèles. Mais le cursus le plus intéressant reste celui d'un quasi-anonyme de Rome ${ }^{65}$, un certain A[el]lius [-], qu'il ne faut plus identifier à Iulius Priscus, le frère de Philippe l'Arabe ${ }^{66}$, et qui se retrouve titulaire de sept procuratèles, toutes de rang ducénaire, ce qui laisse à penser que nous sommes en présence d'un primipile bis, issu de l'ordre équestre.

L'analyse de ces carrières fait ressortir l'extrême variété des nominations; on en retire une impression très nette. A partir du niveau obtenu, tout est possible. Cela revient à dire que le cadre défini officiellement est suffisamment souple pour supporter des nominations répétées dans le même niveau. En effet, on conçoit bien qu'aux niveaux les plus élevés de la hiérachie, c'est-à-dire les procuratèles ducénaires et les officia palatina, il fallait attendre le dégagement des postes pour opérer des promotions. Ceci étant, et dans ce

${ }^{56}$ M. Christol, CCG 9 (1998), 229-235.

${ }^{57}$ Voir les références sous la note 33 .

${ }^{58}$ CIL III $14158=$ C.B. Welles, Gerasa, 449 no. 107, cf. W. Eck Zeitschrift für Papyrologie und Epigraphik 72 (1988), 95-96 (AE 1988, 1054), contra H.-G. Pflaum, SCP nº. 217A.

${ }^{59} \mathrm{AE} 1979,77$.

${ }^{60} \mathrm{AE} 1980,235$.

${ }^{61} \mathrm{R}$. Zucca, 'Un nuovo procurator provinciae Cyrenarum', La Cirenaica in età antica (Macerata, 1995 [1999]), 623-633.

${ }^{62}$ AE 1993, 777 = SEG 42 (1993), 777.

${ }^{63}$ D. Nonnis, Rendiconti della Pontificia Accademi di Archeologica 68 (1995-6 [1999]), 235-262.

${ }^{64}$ G. Alföldy, 'Provincia Hispania superior', Schriften der Philologisch - historischen Klasse der Heidelberger Akademie der Wissenschaten (Heidelberg 2000), 247-262.

${ }^{65}$ CIL VI 1638.

${ }^{66}$ F. Nasti, Zeitschrift für Papyrologie und Epigraphik 117 (1997), 281-290. 
contexte, le jeu multiple des influences, des recommandations et des interventions de toutes sortes faisait que les "règles" officielles n'étaient certainement pas toujours observées. Mais n'en va-t-il pas de même dans beaucoup d'administrations modernes? H.-G. Pflaum avait déjà observé ${ }^{67}$ l'accumulation de postes sexagénaires dans certains cas: preuve d'une part d'une volonté de ne pas promouvoir le fonctionnaire en question, et d'autre part de l'impossibilité de le renvoyer à la vie privée. Sous cet angle, il est aussi très intéressant de considérer les parcours des procurateurs-gouverneurs ${ }^{68}$, même si l'on doit compter avec l'irrésistible ascension des primipiles bis, suivis par les primipilaires.

Certes, avant d'essayer de déterminer l'esprit de l'avancement des carrières, il nous faut bien reconnaitre l'insuffisance de l'information. Trop de débuts restent ignorés; trop souvent, nous ne voyons émerger que les postes les plus importants. Il en va ainsi de T. Petronius Priscus ${ }^{69}$, procurateur ducénaire d'Asie puis de Syrie, avant de devenir l'a libellis d'Hadrien. Cependant, on ne peut arriver qu'à une seule conclusion: la souplesse du système. Le cursus procuratorien est organisé suivant une hiérarchie assez stricte, celle des salaires attachés à la fonction, sur laquelle on ne peut revenir; il est lié à des conditions d'avancement bien précises où l'âge joue son rôle; il est soumis à un calendrier annuel de nomination. Mais ce système supporte sans aucun problème des aménagements, dûs à des facteurs non bureaucratiques, comme la faveur impériale ou la force des recommandations. Ont aussi joué leur rôle les bouleversements dûs aux aléas de l'histoire, comme le remplacement d'une partie du personnel équestre, par exemple lors de la prise de pouvoir de Septime Sévère ou après l'élimination de Plautien ou enfin au moment de l'épuration qui suivit le meurtre de Géta.

Plus l'administration procuratorienne s'amplifia et se développa, plus aussi les structures se rigidifièrent et plus la carrière équestre elle-même s'organisa; c'est l'évolution normale de toute organisation d'Etat. Si on en connaissait mieux les conditions, il est vraisemblable que l'on pourrait faire les mêmes constatations sur les carrières des affranchis impériaux.

On comprend bien alors l'obligation de l'existence d'une structure, même si, dans les faits, elle conservait une grande souplesse: à cet égard, il faut jeter un regard neuf sur les débuts des cursus procuratoriens; les nombreuses

\footnotetext{
${ }^{67}$ Pflaum 1960-1, op.cit. (n.23), $n^{\circ} .280$ : C. Valerius Fuscus.

${ }^{68}$ A. Magioncalda, 'I governatori delle province procuratorie: carriere', L'ordre équestre, histoire d'une aristocratie, (Bruxelles-Louvain 1995 [Rome 1999]), 391-462.

${ }^{69}$ Voir $n .44$ pour la référence.
} 
exceptions qui y apparaissent en témoignent bien. Cette structure était rendue plus nécessaire par le rôle effectif de l'ordre équestre, qui, pour citer $\mathrm{M}$. Christol, ne fut pas "le fidèle servant du palais" ${ }^{10}$ mais un partenaire actif de la transformation du gouvernement de l'Empire.

Paris, Septembre 2000

${ }^{70} \mathrm{M}$. Christol, 'L'ascension de l'ordre équestre. Un thème historiographique et sa réalité', L'ordre équestre, histoire d'une aristocratie (Bruxelles-Louvain 1995 [Rome 1999]), 628. 